\title{
How the point mutation of NPC1 can affect the cholesterol transport efficiency: Molecular dynamics study
}

\author{
Hye-Jin Yoon ${ }^{1 *}$, Soonmin Jang ${ }^{2}$, Hyunah Jeong ${ }^{2}$, and Hyung Ho Lee ${ }^{1}$ \\ ${ }^{1}$ Department of Chemistry, Seoul National University, \\ ${ }^{2}$ Department of Chemistry, Sejong University, \\ yoonhj@snu.ac.kr
}

The NPC1 (Niemann-Pick type C1) is one of the main players of cholesterol control in the lysosome and almost its action is closed combined with NPC2 (Niemann-Pick type C2) protein. The dysfunction of one of the proteins can cause problems in overal chloesterol homestasis and leads to a disease, called the Niemann-Pick type C (NPC) disease. It has been reported that many mutations are responsible to the disease. The point mutation R518W or R518Q on the NPC1 is one of such examples. Even though many details on the cholesterol transport mechanism of NPC1 is elucidated especially with the full-length NPC1 structure obtained from cryo-EM study, it is not obvious how the simple mutation can lead such a big difference in proper function of NPC1. In this respect, the single mutation mentioned above could be a good candidate to relate the dynamical function of NPC1 to its structure in cholesterol transport.

In this presentation, we report how the corresponding mutation can induces the structural change in NPC1 by molecular dynamics simulations. Detailed analysis of the resulting simulation trajectory reveals important structural features that is essential for proper function of the NPC1 for cholesterol transport. It has been found that the mutation leads to structural change that is required for proper interaction with NPC2.

The current study can provide some insights into how the structure is closed related to the function of NPC1 in cholesterol transport in terms of its interaction with NPC2 protein.

[1] Saha, P., et al. (2020) Inter-domain dynamics drive cholesterol transport by NPC1 and NPC1L1 proteins. Elife 9, e57089.

[2] Dubey, V., et al. (2020) Cholesterol binding to the sterol-sensing region of Niemann Pick C1 protein confines dynamics of its N-terminal domain. PLoS Comput Biol 16, e1007554.

Keywords: Molecular dynamics simulation, Niemann-Pick disease type C (NPC) disease 